\title{
Redução do limite de estabilidade direção-específica em indivíduos leve a moderadamente afetados pela doença de Parkinson
}

\author{
Direction-specific limits of stability are reduced in mild to moderate Parkinson's disease \\ individuals
}

Claúdia Diniz ${ }^{1}$, Lidiane Oliveira Lima ${ }^{2}$, Francisco Cardoso $^{3}$, Luci Teixeira-Salmela ${ }^{4}$, Fátima Rodrigues-de-Paula ${ }^{4}$

Estudo desenvolvido no Laboratório de Performance Motora e Funcional Humana do Departamento de Fisioterapia da Universidade Federal de Minas Gerais (UFMG) Belo Horizonte (MG), Brasil.

${ }^{1}$ MSc. Professora da Pontifícia Universidade Católica (PUC) de Minas Gerais - Belo Horizonte (MG), Brasil.

${ }^{2}$ MSc. Doutoranda do Programa de Pós-graduação em Ciências da Reabilitação da UFMG - Belo Horizonte (MG), Brasil.

${ }^{3}$ Ph.D. Professor do Departamento de Clínica Médica da UFMG - Belo Horizonte (MG), Brasil.

${ }^{4}$ Ph.D. Professoras do Departamento de Fisioterapia da UFMG - Belo Horizonte (MG), Brasil.

ENDEREÇO PARA CORRESPONDÊNCIA Fátima Rodrigues-de-Paula Departamento de Fisioterapia da UFMG - Av. Antônio Carlos, 6627 Campus Pampulha - CEP: 31270010 - Belo Horizonte (MG), Brasil - E-mail: fatimarp@globo.com

APRESENTACSÃO mar. 2011

ACEITO PARA PUBLICAÇÃO set. 2011

\section{FONTE DE FINANCIAMENTO}

Conselho Nacional de Desenvolvimento Científico e Tecnológico (CNPq) e Fundação de Amparo à Pesquisa do Estado de Minas Gerais (FAPEMIG)

CONFLITO DE INTERESSE nada a declarar

Parecer de aprovação no Comitê de Ética em Pesquisa da Universidade Federal de Minas Gerais (UFMG) $n^{\circ}$ ETIC 444/05
RESUMO: A instabilidade postural na doença de Parkinson (DP) tem sido associada a uma diminuição do limite de estabilidade (LE) na direção ântero-posterior (AP). Entretanto, ainda que possíveis alterações do LE na direção látero-lateral (LL) tenham sido sugeridas, tal direção não tem sido avaliada nos estudos com DP, principalmente quando o teste de limite de estabilidade (TLE) envolve movimentos intencionais que deslocam o centro de massa corporal (CMC). O objetivo do presente estudo foi investigar o LE na postura de pé durante movimentos voluntários que promovem deslocamento do CMC nas direções AP e LL de indivíduos com e sem a DP. Doze indivíduos com DP (Hoehn \& Yahr=II, III) e 12 sem a doença realizaram o TLE nos sentidos anterior, posterior, direito e esquerdo. A velocidade de movimento (VM), excursão máxima (EM) e o controle direcional (CD) do CMC foram avaliados em cada sentido. Os indivíduos com DP foram significativamente mais lentos em todos os sentidos de deslocamento do CMC $(p<0,05)$. Não houve diferença significativa na EM e CD no sentido anterior entre os grupos $(p>0,05)$. Por outro lado, a EM e CD do $C M C$ foram menores para o grupo DP no sentido posterior $(\mathrm{P})$ e na direção LL $(p<0,05)$. Indivíduos leves a moderadamente afetados pela DP apresentaram redução do LE no sentido P e na direção LL quando comparados ao grupo controle. Os resultados sugerem que tal direção e sentido devam ser treinados em ortostatismo, com movimentos que deslocam voluntariamente o CMC, desde fases iniciais da DP.

Descritores: parkinsonismo; doença de Parkinson; instabilidade postural; controle postural.

ABSTRACT: Postural instability in Parkinson's disease (PD) has been associated with decreases of the limits of stability (LOS) in the anterior-posterior (AP) directions. Although LOS changes in the latero-lateral (LL) directions have been suggested, they have not been evaluated in studies with PD, especially when the LOS tests involve intentional displacements of the body center of mass (COM). The aim of this study was to investigate the LOS in standing posture during voluntary movements that promote movement of the COM in AP and LL directions of individuals with and without PD. Twelve subjects with PD (Hoehn \& Yahr stages of II and III) and 12 without the disease performed the LOS tests in the anterior, posterior, right and left directions. The movement velocity (MV), maximum excursion (ME), and directional control (DC) of the COM were evaluated in all investigated directions. Individuals with PD demonstrated smaller COM displacements in all directions $(p<0.05)$. There were no significant differences between the groups regarding the $M E$ and DC in the anterior direction $(p>0.05)$. However, the ME and DC of the COM were smaller for the PD group in the backward, right, and left directions $(p<0.05)$. Individuals with mild to moderate PD demonstrated decreases in the LOS in the posterior and LL directions, when compared to individuals without the disease. These findings suggested that such directions should be trained in the standing position with voluntary movements of the COM from the early stages of PD.

KeYwords: parkinsonism; Parkinson's disease; postural instability; postural control. 


\section{INTRODUCÃO}

A manutenção do equilíbrio corporal em condições estáticas ou dinâmicas é o principal objetivo do sistema de controle postural ${ }^{1}$. Na posição de pé, o equilíbrio é alcançado quando a projeção vertical do centro de massa corporal (CMC) estiver dentro dos limites da base de suporte ${ }^{2-5}$, região marcada pelo limite de estabilidade $(\mathrm{LE})^{6}$. O LE é a distância máxima que o CMC de uma pessoa pode ser deslocado, durante posturas e transferências funcionais sem alterar a base de suporte, e representa o quanto dessa base o indivíduo consegue utilizar e permanecer estável sem dar um passo ou cair ${ }^{7}$.

O LE tem sido investigado por meio de posturografia, em condições estáticas ou dinâmicas. A condição é estática quando o indivíduo está em pé imóvel sob a plataforma ou quando a plataforma é plana e estacionária ${ }^{5,8}$. A condição é dinâmica quando a plataforma é desnivelada ou móvel $^{8}$ ou quando o indivíduo realiza a máxima inclinação postural voluntária sem alterar a base de suporte ${ }^{9}$. O teste de limite de estabilidade (TLE) é dinâmico e quantifica o LE por meio da distância máxima que o indivíduo consegue movimentar-se intencionalmente, deslocando o CMC sob a base de suporte em diferentes direções, sem perder o equilíbrio ${ }^{10}$. Esse teste simula o que ocorre em situações de transição postural na direção ântero-posterior (AP) tanto no movimento de sentado para de pé ou na direção médio-lateral como em movimentos de alcance em ortostatismo e início da marcha ${ }^{9,11}$. OTLE já foi aplicado em idosos ${ }^{12}$, hemiplégicos ${ }^{13}$ e em indivíduos com doença de Parkinson (DP) $)^{10,14,15}$.

A perda da estabilidade postural na DP foi, tradicionalmente, associada a uma diminuição do LE na direção $A^{9,15-18}$. Entretanto, ainda que possíveis alterações do LE na direção látero-lateral (LL) tenham sido sugeridas ${ }^{19}$, tal direção não tem sido avaliada nos estudos com DP, principalmente quando o TLE envolve movimentos intencionais que deslocam o CMC. Além disso, foi reportado um aumento do risco de quedas em indivíduos com DP na presença de maior oscilação lateral $^{20,21}$ e perante perturbações externas laterais da plataforma de força ${ }^{7,19}$.

O objetivo do presente estudo foi investigar o LE na postura de pé com deslocamento voluntário do CMC nas direções AP e LL de indivíduos com e sem a DP.

\section{METODOLOGIA}

Foi realizado um estudo experimental com um grupo de indivíduos com DP idiopática e um grupo controle sem a doença. A seleção da amostra foi por conveniência, sendo os indivíduos com DP recrutados no Ambulatório de Distúrbios do Movimento da Universidade Federal de Minas Gerais, a partir das consultas médicas realizadas no serviço, e o grupo controle, recrutado na comunidade em geral. Todos assinaram um termo de consentimento aprovado pelo Comitê de Ética local (parecer $n^{\circ} 444 / 05$ ). Participaram do estudo 12 indivíduos com DP, com diagnóstico emitido pelo neurologista do ambulatório, em uso de medicação à base de levodopa, sem hipotensão postural e classificados de acordo com os estágios de Hoehn \& Yahr (HY). Foram excluídos desse grupo os indivíduos com discinesias, submetidos a cirurgias neurológicas, com história ou sintomas de alterações labirínticas ou em uso de medicamento que pudesse interferir no equilíbrio.

Participaram do grupo controle 12 indivíduos sem DP, assintomáticos, capazes de caminhar e ficar de pé independentemente e sem relato de quedas nos últimos 6 meses. Foram excluídos desse grupo os indivíduos com dor de qualquer natureza em época dos testes, com problemas ortopédicos/reumatológicos que interferissem na manutenção da postura ortostática, com hipotensão postural, com problemas visuais, auditivos ou dificuldade de compreensão que impedissem a realização dos testes ou que necessitassem de órtese para permanecer em pé.

Os grupos foram pareados por idade, sexo e Índice de Massa Corporal (IMC).

\section{Procedimentos}

Inicialmente, foram coletados dados de identificação (sexo, idade) e dados antropométricos (altura, peso e IMC) de ambos os grupos. Também foram coletados dados clínicos do grupo DP como tempo de evolução da doença, obtido a partir da data do diagnóstico contida no prontuário do participante e classificação pela escala de HY. Além disso, realizou-se nesse grupo o teste de hipotensão postural, através da comparação dos registros da pressão arterial após 15 minutos de repouso em decúbito dorsal ${ }^{22}$ e no primeiro, segundo e quinto minutos após a passagem para a posição de pé. Uma vez incluídos no estudo, os participantes foram agendados e realizaram o TLE do Balance Master System em um dia subsequente. Entre os indivíduos com DP, os testes foram feitos em torno de uma hora após a ingestão da medicação à base de levodopa.

\section{Teste de limite de estabilidade}

O Balance Master System, versão 8.0.3, é um equipamento composto de duas plataformas unidas por um pino, em cujas extremidades estão quatro sensores de força que detectam pressão. As plataformas são conectadas a um computador com monitor, localizado à frente delas e ao nível dos olhos do indivíduo. O TLE de tal equipamento é um teste dinâmico que quantifica as características de deslocamento do CMC associadas com a capacidade do indivíduo oscilar o corpo voluntariamente em determinadas direções no espaço ${ }^{23}$. Esse teste foi considerado confiável para avaliar o equilíbrio dinâmico com confiabilidade teste-reteste, variando de 0,73 a 0,88 83,24 para as variáveis medidas por ele.

A partir da posição ortostática sobre a plataforma, o indivíduo realiza movimentos voluntários de inclinação do corpo em direção aos alvos que surgem aleatoriamente na tela do computador nos sentidos anterior, posterior, direito e esquerdo. Tais alvos são dispostos na tela de modo circular, em ângulos de $45^{\circ} \mathrm{e}$ posicionados ao nível de dificuldade de $100 \%$ do LE, calculado pelo equipamento com base na altura do participante. A partir da posição central inicial que representa a coincidência do CMC do indivíduo com o centro da plataforma, os participantes foram orientados a se inclinarem em direção a cada alvo, de modo rápido e direto.

A posição ereta inicial do indivíduo sobre a plataforma foi com os braços ao lado do corpo e com os pés posicionados em marcas específicas desenhadas na plataforma. Uma vez informada a altura do indivíduo, o equipamento determinava a distância mantida entre os pés, indicando as marcas de posicionamento específicas para cada um. Se a posição dos pés fosse alterada por qualquer motivo, eles eram 
reposicionados e o teste repetido. A cada lado do indivíduo havia uma pessoa, mantida para ampará-lo, caso ele se desequilibrasse. Foi concedido aos participantes um período de familiarização de até cinco minutos com os movimentos de inclinação em direção aos alvos exigidos durante o teste ${ }^{25}$. O TLE foi realizado 2 vezes no mesmo dia, com intervalo para repouso de aproximadamente 5 minutos ${ }^{25}$, sendo a duração dos dois testes, em média, de 20 minutos.

As variáveis avaliadas foram: velocidade de movimento (VM), excursão máxima (EM) e controle direcional (CD) do CMC. A VM registra a velocidade média do centro de massa computada entre $5 \%$ e 95\% da distância percorrida até a ocorrência do primeiro movimento corretivo da trajetória do CMC, e é expressa em graus por segundo. A EM mede a maior distância percorrida pelo CMC durante cada tentativa de alcançar os alvos. Uma vez que os alvos são posicionados a 100\% do LE, os escores próximos a esse valor são considerados melhores. É expressa em porcentagem do LE. O CD compara a quantidade de movimento intencional em direção ao alvo com a quantidade de movimento corretivo não direcionado ao alvo. É expresso em porcentagem e calculado pelo equipamento a partir da seguinte fórmula: movimento intencional
- movimento corretivo/movimento intencional. Se todo movimento do indivíduo é direcionado ao alvo numa linha reta, a quantidade de movimento corretivo é igual a 0 e o escore é de 100\%. As variáveis foram analisadas nos sentidos anterior (VMA, EMA e CDA), posterior (VMP, EMP e CDP), direito (VMD, EMD e CDD) e esquerdo (VME, EME e CDE), sendo que os sentidos anterior e posterior caracterizaram a direção AP, e os sentidos direito e esquerdo caracterizaram a direção LL.

Foi considerado para análise o melhor escore obtido em cada variável analisada. Estatística descritiva e teste de normalidade (Shapiro-Wilk) foram realizados para todas as variáveis, utilizando o pacote estatístico SPSS ${ }^{\circledR}$, versão 13.0. O teste $t$ de Student para amostras independentes foi utilizado para comparar as variáveis normalmente distribuídas (idade e IMC). Como a maioria das outras variáveis relacionadas à $V M$, à $E M$ e ao $C D$ não apresentou distribuição normal, utilizouse o teste não paramétrico Mann-Whitney para comparação entre o grupo controle e o grupo DP. O nível de significância estabelecido foi de $95 \%$.

\section{RESULTADOS}

O grupo com DP foi composto por quatro mulheres e oito homens que apresentaram tempo médio de duração da doença de 9,0 $0 \pm 3,76$ anos. As médias de idade $(69,67 \pm 6,97$ anos), de altura $(1,62 \pm 0,06 \mathrm{~m})$ e de IMC $(24,74 \pm 3,11)$ do grupo com DP não foram significativamente diferentes das médias das mesmas variáveis do grupo controle, tendo sido o pareamento confirmado pelos testes estatísticos. Entre os indivíduos com DP, nenhum fazia uso de órtese e, de acordo com os Estágios de HY, cinco foram classificados no estágio 2 , um no estágio 2 , e seis no estágio 3.

Os indivíduos com DP apresentaram redução significativa da VM em todos os sentidos de deslocamento do CMC (Tabela 1).

Não houve diferença significativa na variável EMA entre os grupos. Por outro lado, a EM do CMC foi menor para o grupo DP no sentido posterior e direção LL, como observado na Tabela 2. Também não houve diferença significativa entre os grupos para o CDA. Além disso, os indivíduos com DP exibiram valores significativamente menores de $\mathrm{CD}$ do $\mathrm{CMC}$ no sentido posterior e direção LL (Tabela 3).

DISCUSSÃO

No presente estudo, os indivíduos com DP apresentaram menor VM, EM e

Tabela 1. Valores da variável velocidade de movimento do centro de massa corporal em graus/segundo

\begin{tabular}{|c|c|c|c|c|c|c|c|}
\hline & \multicolumn{3}{|c|}{ Controle $(n=12)$} & \multicolumn{3}{|c|}{ Parkinson $(n=12)$} & \multirow{2}{*}{ Valor $p$} \\
\hline & Mínimo & Máximo & Mediana & Mínimo & Máximo & Mediana & \\
\hline VMA & 1,8 & 6,1 & 3,9 & 0,9 & 3,9 & 1,7 & 0,001 \\
\hline VMP & 1,2 & 7,4 & 3,5 & 1,0 & 2,4 & 1,8 & 0,001 \\
\hline VMD & 1,4 & 11,6 & 4,2 & 1,1 & 6,5 & 1,9 & 0,004 \\
\hline VME & 1,9 & 10,5 & 5,9 & 1,2 & 4,4 & 2,3 & 0,014 \\
\hline
\end{tabular}

VMA: velocidade de movimento anterior; VMP: velocidade de movimento posterior; VMD: velocidade de movimento direita; VME: velocidade de movimento esquerda

Tabela 2. Valores da variável excursão máxima do centro de massa corporal em porcentagem do limite de estabilidade

\begin{tabular}{|c|c|c|c|c|c|c|c|}
\hline & \multicolumn{3}{|c|}{ Controle $(n=12)$} & \multicolumn{3}{|c|}{ Parkinson $(n=12)$} & \multirow{2}{*}{ Valor $p$} \\
\hline & Mínimo & Máximo & Mediana & Mínimo & Máximo & Mediana & \\
\hline EMA & 33 & 85 & 69 & 28 & 86 & 44 & 0,056 \\
\hline EMP & 85 & 109 & 98 & 29 & 95 & 73 & 0,000 \\
\hline EMD & 78 & 103 & 96 & 34 & 99 & 72 & 0,003 \\
\hline EME & 83 & 108 & 99 & 47 & 91 & 80 & 0,000 \\
\hline
\end{tabular}

EMA: excursão máxima anterior; EMP: excursão máxima posterior; EMD: excursão máxima direita; EME: excursão máxima esquerda 
Tabela 3. Valores da variável controle direcional do centro de massa corporal em porcentagem do limite de estabilidade

\begin{tabular}{lllllllll}
\hline & \multicolumn{9}{c}{ Controle $(\mathrm{n}=12)$} & \multicolumn{5}{c}{ Parkinson $(\mathrm{n}=12)$} \\
\cline { 2 - 8 } & Mínimo & Máximo & Mediana & Mínimo & Máximo & Mediana & Valor $\mathrm{p}$ \\
\hline CDA & 67 & 94 & 88 & 40 & 94 & 75 & 0,112 \\
CDP & 79 & 96 & 87 & 29 & 91 & 79 & 0,014 \\
CDD & 84 & 96 & 90 & 71 & 91 & 84 & 0,012 \\
CDE & 85 & 95 & 91 & 69 & 93 & 86 & 0,028 \\
\hline
\end{tabular}

CDA: controle direcional anterior; CDP: controle direcional posterior; CDD: controle direcional direito; CDE: controle direcional esquerdo

$C D$, caracterizando uma redução do LE no sentido posterior e direção LL quando comparados ao grupo controle. Estudos prévios demonstraram redução do LE nesses indivíduos a partir da somatória dos valores obtidos em cada sentido ${ }^{10,14,15}$. Essa medida única pode mascarar déficits específicos da direção no LE que comprometam a execução de determinadas atividades funcionais em indivíduos com DP. Por exemplo, a redução do LE na direção $L L$, como demonstrada neste estudo, pode contribuir com as dificuldades para realizar o alcance lateral em ortostatismo e iniciar a marcha nesses indivíduos. Tais atividades exigem o deslocamento do CMC na direção LL para transferir o peso e alcançar um objeto lateralmente ou para elevar o membro inferior contralateral e dar um passo ${ }^{26}$. Da mesma forma, um LE menor no sentido posterior pode comprometer atividades que exijam a inclinação posterior do corpo, como, por exemplo, o alcance de objetos no alto ${ }^{11}$.

Vários estudos avaliaram o LE na direção AP e reportaram uma redução dele em indivíduos com DP $\mathrm{P}^{9,15-17}$. Por outro lado, poucos estudos reportaram o LE na direção LL desses indivíduos ${ }^{17,20}$. Na realidade, os estudos de Mancini et al. ${ }^{17} \mathrm{e}$ de Van Wegen et al. ${ }^{20}$ reportaram a quantidade de oscilação LL do CMC enquanto $\mathrm{O}$ indivíduo inclinava-se na direção $\mathrm{AP}$, o que é funcional e biomecanicamente diferente do deslocamento intencional para os lados, como realizado no presente estudo. Modelos biomecânicos de equilíbrio mostraram que o mecanismo motor que controla o deslocamento do CMC na direção LL é centrado no quadril e tronco, enquanto o controle na direção AP ocorre em torno da articulação do tornozelo ${ }^{4,27,28}$. Assim, os déficits no LE no sentido posterior e direção LL encontrados neste estudo podem ser devido à presença de rigidez e reduzida flexibilidade dos músculos axiais de indivíduos com DP. Além disso, a rigidez presente nos membros inferiores desses indivíduos pode dificultar a flexão necessária para manter o equilíbrio em situação de deslocamento posterior ${ }^{7}$.

É possível que os déficits no LE apresentados por indivíduos com DP, além de serem direção-específicos, estejam também relacionados com a gravidade da doença. Contrariamente ao presente estudo, Mancini et al. ${ }^{17}$ encontraram uma diminuição do LE no sentido anterior de indivíduos nas fases intermediárias e avançadas da DP $(\mathrm{HY}=$ III-IV). Semelhante ao presente estudo, Van Wegen et al. ${ }^{20}$ demonstraram menor LE na direção LL em indivíduos leve a moderadamente comprometidos pela doença $(\mathrm{HY}=\mathrm{II}-\mathrm{III})$ ao serem comparados ao grupo controle. Tais achados sugerem que o menor LE e LL podem estar afetados mais precocemente na evolução da DP, justificando assim uma intervenção nesse sentido. Entretanto, considerando a distinta metodologia usada nos estudos citados, já discutida anteriormente, e a escassez de estudos que avaliaram o LE através do deslocamento intencional do CMC, sugere-se que novos estudos sejam realizados com a mesma metodologia e com indivíduos em diferentes fases da DP.

O grupo com DP foi significativamente mais lento no deslocamento do CMC em todos os sentidos, refletindo um desempenho quase $50 \%$ inferior ao do grupo controle. Resultado semelhante foi obtido por Jessop et al. ${ }^{29}$ também com indivíduos em fases leves a moderadas da DP. A bradicinesia é apontada como a causa da diminuição da VM em diferentes fases da $\mathrm{DP}^{7,14,29}$. Tal sintoma tem sido associado a déficits na produção da potência muscular dos membros inferiores e pode contribuir com quedas recorrentes nesses indivíduos ${ }^{30}$.
Ainda que mais lentamente, o grupo com DP foi similar ao grupo controle quanto à EM e ao $\mathrm{CD}$ do deslocamento no sentido anterior. É possível que os indivíduos em fases leves e moderadas da DP, diferente dos indivíduos sem a doença, priorizem a atenção e a destreza ao invés da velocidade para a execução voluntária do movimento nessa direção ${ }^{29}$. A quantidade similar de movimentos corretivos entre os grupos, demonstrada pela variável CDA (Tabela 3), reforça tal possibilidade.

Este estudo tem algumas limitações. A diminuição do LE encontrada permite inferir déficits do controle postural de indivíduos com DP ao realizarem movimentos intencionais de inclinação corporal a partir da postura de pé, tornando difícil a generalização de tais déficits para outras posturas, como sentado. Além disso, a ausência de medidas de desempenho funcional e de risco de quedas limita a compreensão sobre a relevância clínica do menor LE, bem como sobre a probabilidade de ocorrência de quedas nesses indivíduos. Entretanto, os resultados do presente estudo são valiosos para a prática clínica. As alterações no sentido posterior e direção LL do LE sugerem que tais direções devam ser treinadas com movimentos intencionais por meio de exercícios que enfatizem velocidade, coordenação e distância deslocada do CMC, a fim de minimizar perdas progressivas do controle postural advindas com a DP.

\section{CONCLUSÃO}

Indivíduos leves a moderadamente afetados pela DP apresentaram VM, EM e CD diminuídos, caracterizando uma redução do LE no sentido posterior e direção LL quando comparados ao grupo controle. Os resultados sugerem que tal sentido e direção devam ser treinados em ortostatismo, com movimentos que deslocam voluntariamente o $\mathrm{CMC}$, desde fases iniciais da DP. 


\section{REFERÊNCIAS}

1. Rogind H, Lykkegaard JJ, Bliddal H, Danneskiold-Samsoe B. Postural sway in normal subjects aged 20-70 years. Clin Physiol Funct Imaging. 2003;23(3):171-6.

2. Hof AL, Gazendam MG, Sinke WE. The condition for dynamic stability. J Biomech. 2005;38(1):1-8.

3. Winter DA, Patla AE, Prince F, Ishac M, Gielo-Perczak K. Stiffness control of balance in quiet standing. J Neurophysiol. 1998;80(3):1211-21.

4. Winter DA, Patla AE, Ishac M, Gage WH. Motor mechanisms of balance during quiet standing. J Electromyogr Kinesiol. 2003;13(1):49-56.

5. Duarte M, Freitas SMSF. Revisão sobre posturografia baseada em plataforma de força para avaliação do equilíbrio. Rev bras fisioter. 2010;14(3):183-92.

6. Pollock AS, Durward BR, Rowe PJ, Paul JP. What is balance? Clin Rehabil. 2000; 14(4):402-6.

7. Horak F, Dimitrova D, Nutt J. Direction-specific postural instability in subjects with Parkinson's disease. Exp Neurol. 2005;193(2):504-21.

8. Frenklach A, Louie S, Koop MM, Bronte-Stewart $\mathrm{H}$. Excessive postural sway and the risk of falls at different stages of Parkinson's disease. Mov Disord. 2009;24(3):377-85.

9. Schieppati M, Hugon M, Grasso M, Nardone A, Galante $M$. The limits of equilibrium in young and elderly normal subjects and in parkinsonians. Electroencephalogr Clin Neurophysiol. 1994;93(4):286-98.

10. Rossi M, Soto A, Santos S, Sesar A, Labella T. A prospective study of alterations in balance among patients with Parkinson's Disease. Eur Neurol. 2009;61(3):171-6.

11. Newton RA. Validity of the multi-directional reach test: A practical measure for limits of stability in older adults. J Gerontol A Biol Sci Med Sci. 2001;56(4):M248-52.

12. Melzer I, Benjuya N, Kaplanski J. Postural stability in the elderly: a comparison between fallers and non-fallers. Age Ageing. 2004;33(6):602-7.

13. Tung FL, Yang YR, Lee CC, Wang RY. Balance outcomes after additional sit-to-stand training in subjects with stroke: a randomized controlled trial. Clin Rehabil. 2010;24(6):533-42.

14. Yang YR, Lee YY, Cheng SJ, Lin PY, Wang RY. Relationships between gait and dynamic balance in early Parkinson's disease. Gait Posture. 2008;27(4):611-5.

15. Nallegowda M, Singh U, Handa G, Khanna M, Wadhwa $\mathrm{S}$, Yadav SL, et al. Role of sensory input and muscle strength in maintenance of balance, gait, and posture in Parkinson's disease: a pilot study. Am J Phys Med Rehabil. 2004;83(12):898-908.
16. Menant JC, Latt MD, Menz HB, Fung VS, Lord SR. Postural sway approaches center of mass stability limits in Parkinson's disease. Mov Disord. 2011;26(4):637-43.

17. Mancini M, Rocchi L, Horak FB, Chiari L. Effects of Parkinson's disease and levodopa on functional limits of stability. Clin Biomech. 2008;23(4):450-8.

18. Horak FB, Nutt JG, Nashner LM. Postural inflexibility in parkinsonian subjects. J Neurol Sci. 1992;111(1):46-58.

19. King LA, Horak FB. Lateral stepping for postural correction in Parkinson's disease. Arch Phys Med Rehabil. 2008;89(3):492-9.

20.van Wegen EEH, van Emmerik REA, Wagenaar RC, Ellis T. Stability boundaries and lateral postural control in parkinson's disease. Motor Control. 2001;5(3):254-69.

21. Blaszczyk JW, Orawiec R, Duda-Klodowska D, Opala G. Assessment of postural instability in patients with Parkinson's disease. Exp Brain Res. 2007;183(1):107-14.

22. Senard JM, Rai S, Lapeyre-Mestre M, Brefel C, Rascol O, Rascol A, Montastruc JL. Prevalence of orthostatic hypotension in Parkinson's disease. J Neurol Neurosurg Psychiatry. 1997;63(5):584-9.

23. Neurocom II. Balance Master Operator's Manual. Clackamas: OR; 1999.

24. Liston RA, Brouwer BJ. Reliability and validity of measures obtained from stroke patients using the Balance Master. Arch Phys Med Rehabil. 1996;77(5):425-30.

25. Clark S, Rose DJ. Evaluation of dynamic balance among community-dwelling older adult fallers: a generalizability study of the limits of stability test. Arch Phys Med Rehabil. 2001;82(4):468-74.

26. Martin M, Shinberg M, Kuchibhatla M, Ray L, Carollo J, Schenkman ML. Gait initiation in community-dwelling adults with Parkinson's disease: comparison with older and younger adults without the disease. Phys Ther. 2002;82(6):566-77.

27. Henry SM, Fung J, Horak FB. Control of stance during lateral and anterior/posterior surface translations. IEEE Trans Rehabil Eng. 1998;6(1):32-42.

28. Henry SM, Fung J, Horak FB. Effect of stance width on multidirectional postural responses. J Neurophysiol. 2001;85(2):559-70.

29. Jessop RT, Horowicz C, Dibble LE. Motor learning and Parkinson disease: Refinement of movement velocity and endpoint excursion in a limits of stability balance task. Neurorehabil Neural Repair. 2006;20(4):459-67.

30. Allen NE, Sherrington C, Canning CG, Fung VSC. Reduced muscle power is associated with slower walking velocity and falls in people with Parkinson's disease. Parkinsonism Relat Disord. 2010;16(4):261-4. 